\title{
Social Network Analysis using RLVECN: Representation Learning via Knowledge-Graph Embeddings and Convolutional Neural-Network
}

\author{
Bonaventure C. Molokwu* , Ziad Kobti \\ School of Computer Science, University of Windsor, Windsor - Ontario, Canada \\ \{molokwub, kobti\}@uwindsor.ca
}

\begin{abstract}
Social Network Analysis (SNA) has become a very interesting research topic with regard to Artificial Intelligence (AI) because a wide range of activities, comprising animate and inanimate entities, can be examined by means of social graphs. Consequently, classification and prediction tasks in SNA remain open problems with respect to AI. Latent representations about social graphs can be effectively exploited for training AI models in a bid to detect clusters via classification of actors as well as predict ties with regard to a given social network. The inherent representations of a social graph are relevant to understanding the nature and dynamics of a given social network. Thus, our research work proposes a unique hybrid model: Representation Learning via Knowledge-Graph Embeddings and ConvNet (RLVECN). RLVECN is designed for studying and extracting meaningful representations from social graphs to aid in node classification, community detection, and link prediction problems. RLVECN utilizes an edge sampling approach for exploiting features of the social graph via learning the context of each actor with respect to its neighboring actors.
\end{abstract}

\section{Introduction}

Human habitat is comprised of several systems and ecosystems; and interaction is a natural phenomenon obtainable in any given system or ecosystem. Interaction between constituent entities in a given system/ecosystem is a strategy for survival, and essential for the sustenance of the system/ecosystem. Social graphs are non-static structures. Analyzing and learning underlying knowledge from communities, comprising social actors, using given sets of standard still remain a crucial research problem in SNA. Hence, we have proposed RLVECN which is a hybrid model for classificationbased and prediction-related problems in social networks.

On one hand, the classification of nodes induces the formation of cluster(s). Consequently, clusters give rise to homophily in social networks. On the other hand, the prediction of links brings about correlations and/or ties formation;

\footnotetext{
${ }^{*}$ Contact Author
}

which increases the tendency for transitivity in social graphs. RLVECN is based on an iterative learning approach. RLVECN aims at solving the problems of node classification, community detection, and link prediction in SNA using an edge sampling strategy. Basically, learning in RLVECN is effectuated via semi-supervised training. The architecture of RLVECN comprises two (2) distinct representation-learning layers, viz: an embedding layer and a Convolutional Neural Network (ConvNet) layer [Molokwu, 2019]; which are trained by means of unsupervised learning. These layers are essentially featureextraction and dimensionality-reduction layers where viable facts are automatically extracted from the social networks [Molokwu and Kobti, 2019]. The embedding layer projects the feature representation of the social graph to a q-dimensional real-number space, $\mathbb{R}^{q}$. This is accomplished by associating a real (number) vector to every unique actor in the social network; such that the cosine distance of any given tie (a pair of actors) would capture a significant degree of correlation between the two associated actors or nodes. Additionally, the ConvNet layer feeds on the embedding layer; and it is responsible for further extraction of inherent features from the social graph. With reference to RLVECN's architecture, a classification layer succeeds the representation-learning layers; and this layer is trained by means of supervised learning. The classifier is based on a Neural Network (NN) architecture assembled using deep (multi) layers of stacked perceptrons [Goodfellow et al., 2017]. Every low-dimensional feature $(X)$, extracted by the representation-learning layers, is mapped to a corresponding output label $(Y)$; and these $(X, Y)$ pairs are used to supervise the training of the classifier such that it can effectively and efficiently learn how to classify actors, identify clusters, and predict links within the given social graph.

Furthermore, we have evaluated RLVECN against an array of state-of-the-art methodologies and Representation Learning (RL) models which serve as our baselines. Thus, the baselines used herein with respect to the node-classification tasks are:

(i) DeepWalk: Online Learning of Social Representations [Perozzi et al., 2014].

(ii) GCN: Semi-Supervised Classification with Graph Convolutional Networks [Kipf and Welling, 2017].

(iii) LINE: Large-scale Information Network Embedding [Tang et al., 2015].

(iv) Node2Vec: Scalable Feature Learning for Networks 
[Grover and Leskovec, 2016].

(v) SDNE: Structural Deep Network Embedding [Wang et al., 2016].

Also, the baselines used herein for the link-prediction tasks:

(i) ComplEx: Complex Embeddings for Simple Link Prediction [Trouillon et al., 2016] [Lacroix et al., 2018].

(ii) ConvKB: A Novel Embedding Model for Knowledge Base Completion Based on Convolutional Neural Network [Nguyen et al., 2018].

(iii) DistMult: Embedding Entities and Relations for Learning and Inference in Knowledge Bases [Yang et al., 2015].

(iv) HolE: Holographic Embeddings of Knowledge Graphs [Nickel et al., 2016].

\section{Proposed Methodology and Framework}

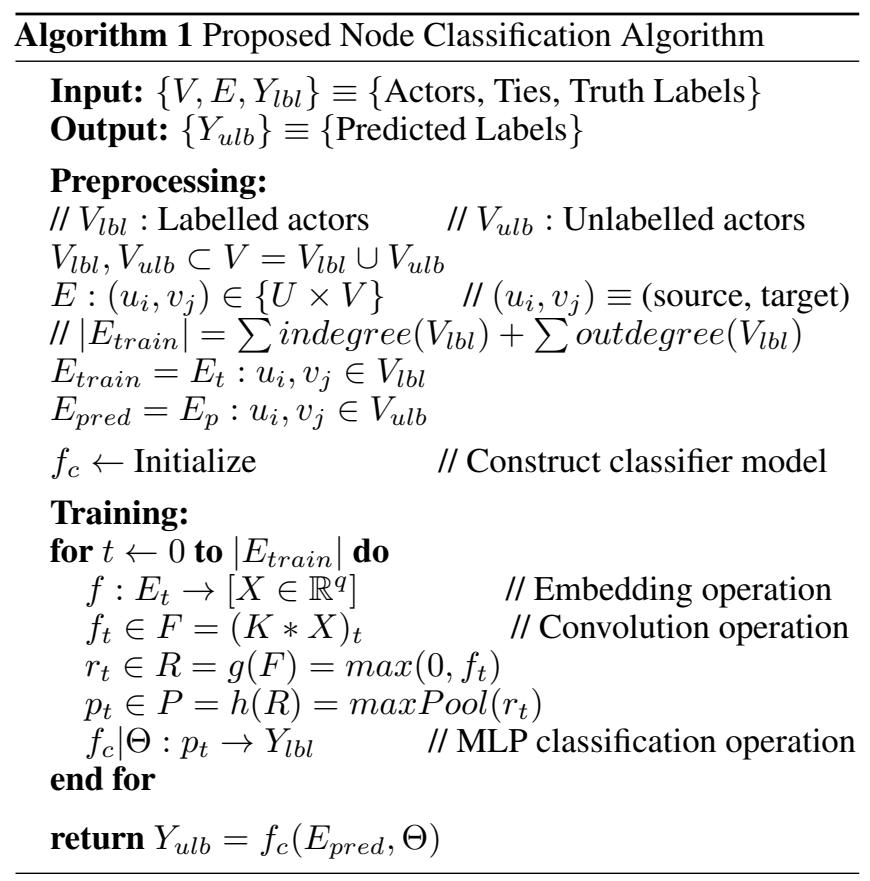

\section{Experiments, Discussions, and Conclusion}

We have modelled the open problems in SNA solved herein as classification problems. Hence, our results are based on a range of classification-based objective functions. Thus, Categorical Cross Entropy was employed as the cost/loss function; while the fitness/utility was measured based on the following metrics: Precision (PC), Recall (RC), F1-score (F1), Accuracy (AC), and Area Under the Receiver Operating Characteristic Curve (RO). The Support (SP) represents the number of ground-truth samples per class for each benchmark dataset.

RLVECN's surpassing performance, in comparison with the baselines benchmarked herein, is primarily attributed to its dual RL layers which enable it to extract and learn sufficient features of each social network representation. Conclusively, its biform RL kernel places it at an edge above the state-ofthe-art baselines as discovered/reported via our experiments.

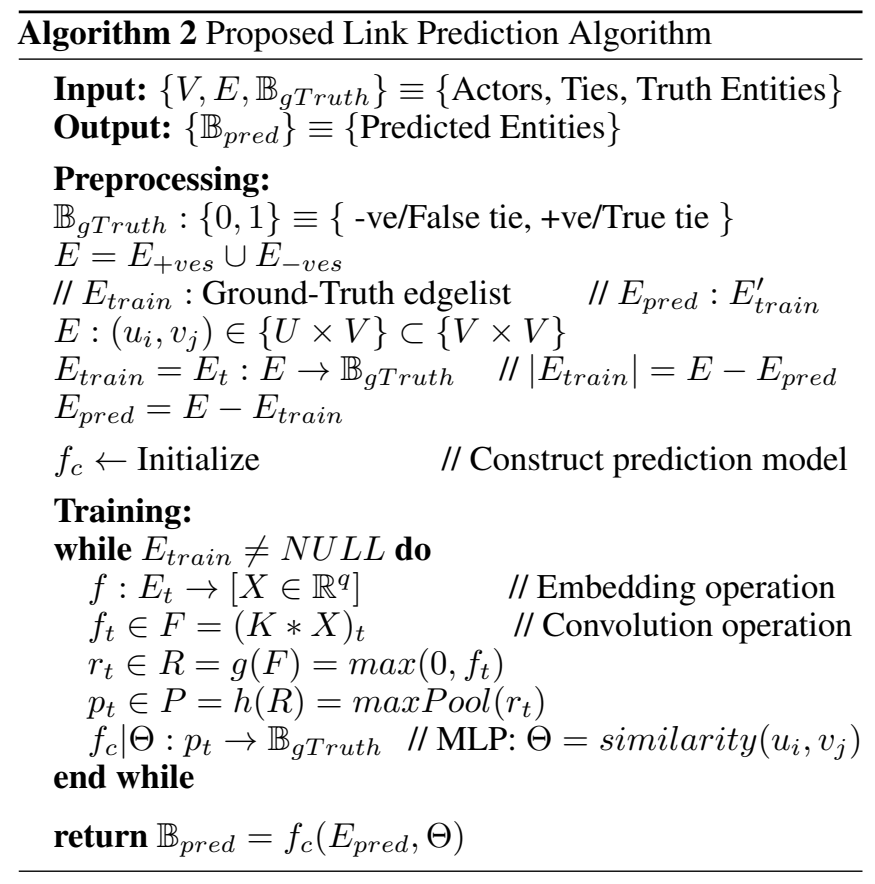

\section{Acknowledgments}

This research was supported by International Business Machines (IBM), SHARCNET and Compute Canada.

\section{References}

[Goodfellow et al., 2017] Ian G. Goodfellow, Yoshua Bengio, and Aaron C. Courville, editors. Deep Learning. MIT Press, Cambridge, MA, 2017.

[Grover and Leskovec, 2016] Aditya Grover and Jure Leskovec. node2vec: Scalable feature learning for networks. Proceedings of the 22nd ACM SIGKDD International Conference, 2016:855-864, 2016.

[Kipf and Welling, 2017] Thomas N. Kipf and Max Welling. Semi-supervised classification with graph convolutional networks. ICLR, 2017.

[Lacroix et al., 2018] Timothée Lacroix, Nicolas Usunier, and Guillaume Obozinski. Canonical tensor decomposition for knowledge base completion. Proceedings of the 35th ICML, abs/1806.07297, 2018.

[Molokwu and Kobti, 2019] Bonaventure C. Molokwu and Ziad Kobti. Event prediction in complex social graphs via feature learning of vertex embeddings. In Neural Information Processing. Springer Publishing, 2019.

[Molokwu, 2019] Bonaventure C. Molokwu. Event prediction in complex social graphs using one-dimensional convnet. In Proceedings of the 28th IJCAI, 2019.

[Nguyen et al., 2018] Dai Q. Nguyen, Tu D. Nguyen, Dat Q. Nguyen, and Dinh Q. Phung. A novel embedding model for knowledge base completion based on convolutional neural network. In Proceedings of the 16th NAACL-HLT, 2018. 